\title{
Is intrauterine exposure to acetaminophen associated with emotional and hyperactivity problems during childhood? Findings from the 2004 Pelotas birth cohort
}

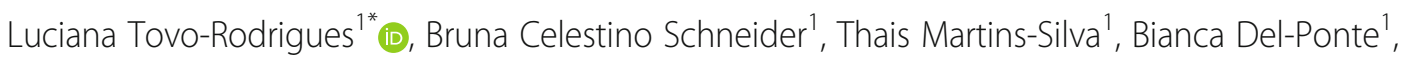
Christian Loret de Mola ${ }^{2}$, Lavinia Schuler-Faccini ${ }^{3}$, Fernanda Sales Luiz Vianna ${ }^{4,5,6}$, Tiago N. Munhoz ${ }^{1,7}$, Ludmila Entiauspe ${ }^{1}$, Mariângela Freitas Silveira ${ }^{1}$, Iná S. Santos ${ }^{1}$, Alicia Matijasevich ${ }^{8}$, Aluísio J. D. Barros ${ }^{1}$, Luis Augusto Rohde ${ }^{9,10}$ and Andréa Dâmaso Bertoldi ${ }^{1}$

\begin{abstract}
Background: Longitudinal studies have consistently reported that prenatal exposure to acetaminophen can to lead to an increased risk of attention deficit-hyperactivity disorder during childhood. This study aimed to investigate the association between intrauterine exposure to acetaminophen and the presence of emotional and behavioral problems at the ages of 6 and 11 years in a low-middle income country.
\end{abstract}

Methods: We performed a prospective longitudinal population-based study using data from the 2004 Pelotas birth cohort. From the 4231 initial cohort participants, 3722 and 3566 children were assessed at 6 and 11 years of age, respectively. The outcomes were assessed using the parent version of Strengths and Difficulties Questionnaire (SDQ). The cut-off points established for the Brazilian population were used to categorize the outcomes. Crude and adjusted odds ratio were obtained through logistic regression.

Results: Acetaminophen was used by $27.5 \%$ (95\% confidence interval [CI]: 26.1-28.9) of the mothers at least once during pregnancy. The prevalence of emotional problems at 6 and 11 years was 13.6 and 19.9\%, respectively. For hyperactivity problems, prevalence was 13.9 and $16.1 \%$, respectively. Intrauterine exposure to acetaminophen increased the odds of having emotional (odds ratio $[\mathrm{OR}]=1.47 ; 95 \% \mathrm{Cl}$ : 1.07-2.02) and hyperactivity/inattention (OR $=1.42 ; 95 \%$ $\mathrm{Cl}$ : 1.06-1.92) problems in 6-year-old boys. At the age of 11, a small decrease in the effect was observed for both outcomes after adjustment: $\mathrm{OR}=1.31$ (95\% Cl: 0.99-1.73) for emotional problems and $\mathrm{OR}=1.25$ (95\% Cl: 0.95-1.65) for hyperactivity/inattention in boys. No association for any phenotypes at both ages was observed for girls.

Conclusion: The effect of intrauterine exposure to acetaminophen in emotional and hyperactivity symptoms was dependent on sex in a Brazilian cohort. While it seemed to be important for boys, mainly at 6 years of age, for girls, no association was observed.

Keywords: Acetaminophen (paracetamol), Prenatal exposure, Birth cohort, Behavioral symptoms

\footnotetext{
* Correspondence: luciana.tovo@gmail.com

${ }^{1}$ Postgraduate Program in Epidemiology, Universidade Federal de Pelotas,

Rua Marechal Deodoro, 1160 - $3^{\circ}$ andar, Pelotas, RS 96020-220, Brazil

Full list of author information is available at the end of the article
}

(c) The Author(s). 2018 Open Access This article is distributed under the terms of the Creative Commons Attribution 4.0 International License (http://creativecommons.org/licenses/by/4.0/), which permits unrestricted use, distribution, and reproduction in any medium, provided you give appropriate credit to the original author(s) and the source, provide a link to the Creative Commons license, and indicate if changes were made. The Creative Commons Public Domain Dedication waiver (http://creativecommons.org/publicdomain/zero/1.0/) applies to the data made available in this article, unless otherwise stated. 


\section{Background}

Acetaminophen (or paracetamol) is one of the most commonly used analgesics worldwide [1]. It is the first choice for pain and fever medication among pregnant women [2]. In Brazil, studies reporting acetaminophen use during pregnancy are scarce [3-5]. These studies are restricted to convenience samples, and to our knowledge, there are no population-based studies that have evaluated its use.

Several birth cohort studies have reported that prenatal exposure to acetaminophen is associated with neurobehavioral and neurodevelopmental disorders during childhood [6-12]. Intrauterine exposure to acetaminophen has been associated with (i) attention-deficit/ hyperactivity disorder (ADHD) [8-12]; (ii) hyperactivity/ inattention and behavioral symptoms [7-11]; (iii) autism spectrum disorder (ASD) [6-10]; and (iv) impaired motor development, difficulty in communication and behavioral disorders [9-13]. In a recent meta-analysis, acetaminophen exposure during pregnancy was associated with a $20-30 \%$ increase in the risk of neurodevelopmental disorders [14]. Only one study has evaluated behavioral outcomes at two different time points during childhood [7].

Acetaminophen crosses the human placental barrier, and in vitro and in vivo findings provide additional evidence in support of observational epidemiological studies. Acetaminophen use has been shown to interfere with neurotransmitter, endocrine and immune systems, as well as with the regulation of brain-derived neurotrophic factor and cell oxidative stress, which are processes associated with brain development [15-22].

Although several variables have been included as covariates in previous studies, the results are susceptible to potential confounding effects [14], which makes it difficult to ascertain whether the use of acetaminophen is safe during pregnancy and indicates the need for more studies in the field. It is of note that current observational studies have been conducted in only European populations [23-25]. In this sense, studies in other populations, such as those from low and medium-income countries, would contribute to the knowledge in the literature and improve the current understanding of the risk of intrauterine exposure to acetaminophen.

This study aims to investigate the association between intrauterine exposure to acetaminophen and the presence of emotional and behavioral symptoms at the ages of 6 and 11 years in the 2004 Pelotas birth cohort.

\section{Methodology}

\section{Target population}

The present investigation is a prospective longitudinal study using data from the 2004 Pelotas birth cohort, a population-based study that documented hospital childbirths in the southern Brazilian city of Pelotas. Among the 4263 live births, 4231 were considered eligible and were included in the present study. So far, these children have been visited at the ages of 3, 12, 24, 48 and 72 months and 11 years, with follow-up rates of 95.7, 94.3, 93.5, 92.0, 90.2 and $86.6 \%$, respectively $[26,27]$. The present study used data from perinatal assessments and follow-ups at ages 6 and 11. At both follow-ups, the children underwent a wide-ranging health assessment with interviews [26].

\section{Use of acetaminophen during pregnancy}

A standardized questionnaire was used during the perinatal evaluation conducted after the birth of the children. The mother's use of medication during pregnancy was retrospectively framed as the following question: "Did you use any medications during pregnancy?" For positive answers, the mothers were asked to report the names of all medicines used during pregnancy, as well as the beginning and end of use. Our exposure variable was defined as medication composed of acetaminophen that was used at least once during pregnancy.

\section{Outcome measurements}

Behavioral symptoms were evaluated using the standardized scores from the Strengths and Difficulties Questionnaire (SDQ). The SDQ is a screening questionnaire, which measures 25 psychological attributes divided into 5 scales: inattention/hyperactive symptoms, conduct problems, emotional symptoms, peer relationship problems, and prosocial behavior. All attributes except prosocial behavior are summed to create a total difficulties score. The questionnaire was adapted and previously validated for the Brazilian population of children and teenagers aged between 4 and 16 years [28].

Trained psychologists administered the SDQ in a standardized manner to the parents or caregivers during each follow-up. The cut-off points established for the Brazilian population were used to categorize the general difficulties scale and the subscales as follows: $\geq 17$ for the total difficulties score, $\geq 5$ for the emotional problems score, $\geq 4$ for the conduct problems score, $\geq 7$ for the inattention/hyperactivity score, $\geq 4$ for the peer problems score, and $\leq 4$ for the prosocial score.

\section{Confounding factors}

The analyses included variables associated with psychiatric disorders and differential use of acetaminophen as follows: National Economic Index score (an economic indicator extensively used in Brazil based on information from the 2000 demographic census by the Brazilian Institute for Geography and Statistics) [29]; sex (male, female); mother's educational level (years of school attendance); mother's age (years); mother's skin color (white, black or other); mother's parity (number of 
children), smoking activity during pregnancy ("Did you smoke during pregnancy?"; yes or no); alcohol consumption during pregnancy ("Did you drink alcoholic beverages during pregnancy?"; yes or no); mood issues during pregnancy ("During pregnancy, did you have depression or suffer from anxiety?"; "no", "yes, not treated" or "yes, treated" and categorized into yes or no responses); infections during pregnancy (yes or no); prepregnancy body mass index (BMI); and other nonsteroidal analgesic use during pregnancy (yes or no).

\section{Statistical analyses}

Statistical analyses were performed using Stata software (version 12.1). The analyses included children who had information available regarding maternal use of acetaminophen during pregnancy and who were assessed using the SDQ at the ages of 6 and 11 years $(N=3470$ and $N=$ 3447 , respectively). The crude and adjusted analyses between exposure to acetaminophen and the outcomes were performed using logistic regression. Specific effects of acetaminophen in boys and girls were also tested.

As a sensitivity analysis, to test the specificity of acetaminophen compared to other nonsteroidal analgesics, a variable regarding the use of other analgesics was created and used as a comparative exposure. All models using intrauterine acetaminophen exposure were also adjusted for this variable. Moreover, to assess the extent of the imprecision in the mood problem information (collected perinatally), we used information from the Edinburgh Postnatal Depression Scale (EPDS) [30], which has been validated for Brazilian populations [31], as a sensitivity analysis. Data were gathered when the child was 12 months old, as this time point it correlates highly with the mood problem information collected at the perinatal interview. The cut-off of 10 points, which is the recommended cut-off for screening in the Brazilian population [31], was used. The EPDS information was added as a covariable in the models, thus replacing the variable that was gathered perinatally.

\section{Results}

Acetaminophen was used during pregnancy at a frequency of $27.5 \%$ (95\% confidence interval [CI]: 26.1-28.9). The prevalence of behavioral disorders assessed through the total difficulties scale of the SDQ was 10.8\% (95\% CI: 9.711.8 ) at the age of 6 and $13.9 \%$ (95\% CI: $12.8-15.1)$ at the age of 11 (Table 1). The sociodemographic, behavioral, and maternal health characteristics are also shown in Table 1. The associations between the covariables and both the exposure and the main outcomes of the study are shown in Additional file 1: Table S1.

Crude and adjusted analyses for the total sample are presented in Table 2. No associations between acetaminophen exposure and total problems or SDQ subscales were observed after adjustment. However, a significant interaction effect was observed for the emotional $(p=0.046)$ and hyperactivity/inattention $(p=0.018)$ subscales at 6 years old. Further analyses were conducted and stratified according to the sex of the children.

Table 3 presents the association between prenatal exposure to acetaminophen and the outcomes evaluated in 6 -year-old boys and girls. At this age, for boys, the use of acetaminophen during pregnancy was associated with emotional (OR $=1.47 ; 95 \% \mathrm{CI}: 1.07-2.02)$, as well as with hyperactivity/inattention ( $\mathrm{OR}=1.42$; 95\% CI: $1.06-1.92)$, problems in the adjusted model. The use of acetaminophen during pregnancy was not associated with other outcomes. For girls, no association was observed.

The effect sizes in the assessment at 11 years of age were slightly smaller than those of the 6 year-old assessment, and most of the associations that were observed at 6 years did not reach significance at 11 years (Table 4 ). Among boys, the crude analysis showed an effect for emotional problems $(\mathrm{OR}=1.33$; 95\% CI: 1.03-1.71). However, this effect lost significance after adjustment $(\mathrm{OR}=1.31$; 95\% CI: 0.99-1.73). Regarding the hyperactivity/inattention subscale, the estimates for crude and adjusted models did not reach statistical significance. Acetaminophen use during pregnancy was not associated with other outcomes for either boys or girls in the adjusted model.

\section{Sensitivity analyses}

To test the specificity of the effect of acetaminophen exposure during intrauterine life on the outcomes of emotional symptoms and hyperactivity, we performed another analysis to test the effect of other nonsteroidal analgesics on the same outcomes. No associations between nonsteroidal analgesics and emotional symptoms $\left(\mathrm{OR}_{\text {adjusted }}=1.09 ; 95 \% \mathrm{CI}: 0.77-1.55\right)$ or hyperactivity symptoms $\left(\mathrm{OR}_{\text {adjusted }}=1.07 ; 95 \% \mathrm{CI}: 0.77-1.48\right)$ were found. No association was observed for either sex for any of the other outcomes (results not shown). In addition, nonsteroidal analgesic use was not associated with any outcomes at 11 years of age.

The second sensitivity analysis aimed to test the imprecision of reported mood problems during pregnancy that were collected perinatally. We performed this analysis by replacing the mood problems variable during pregnancy with the maternal depression information assessed with EPDS in the statistical models. Adjusted analysis results are presented in Table 5. Analyses did not change substantially. The same associations as before were observed in the 6 year-old assessment. In the 11-year-old assessment, the magnitude of the effect of acetaminophen on emotional problems in boys was greater than that in the previous analysis, and the adjusted analysis became significant $(\mathrm{OR}=1.33 ; 95 \% \mathrm{CI}$ : 1.01-1.76). 
Table 1 Sociodemographic characteristics, maternal health, and use of acetaminophen and other analgesics during pregnancy among mothers of boys and girls in the 2004 Pelotas birth cohort who were assessed by SDQ at the follow-ups at 6 and 11 years of age

\begin{tabular}{|c|c|c|c|c|}
\hline \multirow[t]{2}{*}{ Maternal characteristics } & \multicolumn{2}{|c|}{ Follow-up at the age of 6 years } & \multicolumn{2}{|c|}{ Follow-up at the age of 11 years } \\
\hline & $\begin{array}{l}\text { Boys } \\
(N=1812) \\
N(\%)\end{array}$ & $\begin{array}{l}\text { Girls } \\
(N=1658) \\
N(\%)\end{array}$ & $\begin{array}{l}\text { Boys } \\
(N=1781) \\
N(\%)\end{array}$ & $\begin{array}{l}\text { Girls } \\
(N=1666) \\
N(\%)\end{array}$ \\
\hline \multicolumn{5}{|l|}{ Age (years) } \\
\hline$\leq 24$ & $818(45.14)$ & $761(45.93)$ & $793(44.53)$ & $750(45.07)$ \\
\hline $25-29$ & $416(22.96)$ & $370(22.33)$ & $402(22.57)$ & $374(22.48)$ \\
\hline $30-34$ & $388(21.41)$ & $343(20.70)$ & $393(22.07)$ & $355(21.33)$ \\
\hline$\geq 35$ & $190(10.49)$ & $183(11.04)$ & $193(10.84)$ & $185(11.12)$ \\
\hline \multicolumn{5}{|l|}{ Educational level (years) } \\
\hline $0-4$ & $268(14.91)$ & $250(15.26)$ & $257(14.54)$ & $248(15.07)$ \\
\hline $5-8$ & $753(41.88)$ & $697(42.55)$ & $726(41.09)$ & $692(42.04)$ \\
\hline $9-11$ & $589(32.76)$ & $546(33.33)$ & 599 (33.90) & $560(34.02)$ \\
\hline$\geq 12$ & $188(10.46)$ & $145(8.85)$ & $185(10.47)$ & $146(8.87)$ \\
\hline \multicolumn{5}{|l|}{ National Economic Index } \\
\hline 1 (higher) & $341(18.82)$ & $311(18.76)$ & $325(18.25)$ & $302(18.13)$ \\
\hline 2 & $362(19.98)$ & $366(22.07)$ & 355 (19.93) & $363(21.79)$ \\
\hline 3 & $357(19.70)$ & $342(20.63)$ & $348(19.54)$ & $349(20.95)$ \\
\hline 4 & $389(21.47)$ & $329(19.84)$ & $386(21.67)$ & $337(20.23)$ \\
\hline 5 (lower) & $363(20.03)$ & $310(19.70)$ & $367(20.61)$ & $315(18.91)$ \\
\hline \multicolumn{5}{|l|}{ Skin color } \\
\hline White & $1125(62.92)$ & $1016(61.80)$ & $1106(62.98)$ & $1015(61.52)$ \\
\hline Black & $298(16.67)$ & $276(16.79)$ & $291(16.57)$ & $275(16.67)$ \\
\hline Other $^{a}$ & $365(20.41)$ & $352(21.41)$ & $359(20.44)$ & $360(21.82)$ \\
\hline \multicolumn{5}{|c|}{ Smoking during pregnancy } \\
\hline No & $1332(73.51)$ & $1202(72.50)$ & $1320(74.12)$ & $1212(72.75)$ \\
\hline Yes & $480(26.49)$ & $456(27.50)$ & $461(25.88)$ & $454(27.25)$ \\
\hline \multicolumn{5}{|c|}{ Use of alcohol during pregnancy } \\
\hline No & $1750(96.58)$ & $1605(96.80)$ & $1720(96.57)$ & $1616(97.00)$ \\
\hline Yes & $62(3.42)$ & $53(3.20)$ & $61(3.43)$ & $50(3.00)$ \\
\hline \multicolumn{5}{|l|}{ Parity } \\
\hline 1 & $751(41.45)$ & $623(37.60)$ & $740(41.55)$ & $618(37.12)$ \\
\hline 2 & $477(26.32)$ & $443(26.74)$ & $472(26.50)$ & $454(27.27)$ \\
\hline 3 or more & $584(32.23)$ & $591(35.67)$ & 569 (31.95) & $593(35.62)$ \\
\hline \multicolumn{5}{|l|}{ Pre-gestational BMI } \\
\hline Underweight & $75(4.49)$ & $69(4.54)$ & $75(4.59)$ & $66(4.32)$ \\
\hline Normal weight & $1025(61.34)$ & 909 (59.76) & 996 (60.95) & $914(59.82)$ \\
\hline Overweight & $402(24.06)$ & $350(23.01)$ & $401(24.54)$ & $358(23.43)$ \\
\hline Obese & $169(10.11)$ & $193(12.69)$ & $162(9.91)$ & $190(12.43)$ \\
\hline \multicolumn{5}{|c|}{ Mood symptoms during pregnancy } \\
\hline No & $1362(75.17)$ & $1254(75.72)$ & $1351(75.86)$ & $1268(76.20)$ \\
\hline Yes & $450(24.83)$ & $402(24.28)$ & $430(24.14)$ & $396(23.80)$ \\
\hline \multicolumn{5}{|c|}{ Infections during pregnancy } \\
\hline No & $1066(58.96)$ & $974(58.89)$ & $1061(59.71)$ & $975(58.63)$ \\
\hline Yes & $742(41.04)$ & $680(41.11)$ & 716 (40.29) & $688(41.37)$ \\
\hline
\end{tabular}


Table 1 Sociodemographic characteristics, maternal health, and use of acetaminophen and other analgesics during pregnancy among mothers of boys and girls in the 2004 Pelotas birth cohort who were assessed by SDQ at the follow-ups at 6 and 11 years of age (Continued)

\begin{tabular}{|c|c|c|c|c|}
\hline \multirow[t]{2}{*}{ Maternal characteristics } & \multicolumn{2}{|c|}{ Follow-up at the age of 6 years } & \multicolumn{2}{|c|}{ Follow-up at the age of 11 years } \\
\hline & $\begin{array}{l}\text { Boys } \\
(N=1812) \\
N(\%)\end{array}$ & $\begin{array}{l}\text { Girls } \\
(N=1658) \\
N(\%)\end{array}$ & $\begin{array}{l}\text { Boys } \\
(N=1781) \\
N(\%)\end{array}$ & $\begin{array}{l}\text { Girls } \\
(N=1666) \\
N(\%)\end{array}$ \\
\hline \multicolumn{5}{|l|}{ Use of acetaminophen during pregnancy } \\
\hline Never & $1287(71.03)$ & $1218(73.46)$ & $1259(70.69)$ & $1229(73.77)$ \\
\hline At least once & $525(28.97)$ & $440(26.54)$ & $522(29.31)$ & $437(26.23)$ \\
\hline \multicolumn{5}{|l|}{ Use of other analgesics during pregnancy } \\
\hline Never & $1430(79.53)$ & $1310(79.25)$ & $1402(79.21)$ & $1311(78.83)$ \\
\hline At least once & $368(20.47)$ & $343(20.75)$ & $368(20.79)$ & $352(21.17)$ \\
\hline \multicolumn{5}{|l|}{ Outcomes } \\
\hline Total SDQ (score $\geq 17$ pts) & $212(11.70)$ & $161(9.71)$ & $288(16.17)$ & $192(11.52)$ \\
\hline Emotional symptoms (score $\geq 5$ pts) & $237(13.08)$ & $235(14.17)$ & $339(19.03)$ & $347(20.83)$ \\
\hline Conduct Problems (score $\geq 4$ pts) & $295(16.28)$ & $220(13.27)$ & $253(14.21)$ & $198(11.88)$ \\
\hline Hyperactivity/inattention (score $\geq 7$ pts) & $288(15.89)$ & $193(11.64)$ & $367(20.61)$ & 189 (11.34) \\
\hline Peer relationship problems (score pts) & $277(15.29)$ & $213(12.85)$ & $269(15.10)$ & $190(11.40)$ \\
\hline Prosocial behavior (score $\leq 4 \mathrm{pts}$ ) & $23(1.27)$ & $18(1.09)$ & $28(1.57)$ & $20(1.20)$ \\
\hline
\end{tabular}

Other skin color: self-classification as brown, yellow or indigenous

Table 2 Association between use of acetaminophen by the mother during pregnancy and mental health outcomes at the age of 6 years $(N=3470)$

\begin{tabular}{|c|c|c|c|c|c|c|}
\hline \multirow[t]{2}{*}{ Outcomes } & \multicolumn{2}{|c|}{$\begin{array}{l}\text { Frequency of outcome according to use } \\
\text { of acetaminophen during pregnancy } N(\%)\end{array}$} & \multirow{2}{*}{$\begin{array}{l}\text { Crude analysis } \\
\text { OR } \\
(95 \% \mathrm{Cl})\end{array}$} & \multicolumn{2}{|c|}{ Adjusted analysis $^{a}$} & \multirow{2}{*}{$\begin{array}{l}p \text {-value for sex - use } \\
\text { of acetaminophen } \\
\text { interaction term }^{b}\end{array}$} \\
\hline & $\begin{array}{l}\text { Exposed } \\
\mathrm{n}(\%)\end{array}$ & $\begin{array}{l}\text { Not exposed } \\
\mathrm{n}(\%)\end{array}$ & & $\begin{array}{l}\mathrm{OR} \\
(95 \% \mathrm{Cl})\end{array}$ & $p$ & \\
\hline \multicolumn{7}{|l|}{6 years old } \\
\hline Total SDQ (score $\geq 17$ pts) & $102(10.57)$ & $271(10.82)$ & $0.97(0.77-1.24)$ & $1.15(0.88-1.50)$ & 0.313 & 0.454 \\
\hline Emotional symptoms (score $\geq 5$ pts) & $137(14.20)$ & $335(13.37)$ & $1.07(0.87-1.33)$ & $1.15(0.91-1.46)$ & 0.232 & 0.046 \\
\hline Conduct problems (score $\geq 4$ pts) & $119(12.33)$ & $396(15.81)$ & $0.75(0.60-0.93)$ & $0.86(0.68-1.10)$ & 0.222 & 0.572 \\
\hline $\begin{array}{l}\text { Hyperactivity/inattention } \\
\text { (score } \geq 7 \text { pts) }\end{array}$ & $137(14.20)$ & $344(13.73)$ & $1.04(0.84-1.29)$ & $1.10(0.87-1.39)$ & 0.427 & 0.018 \\
\hline $\begin{array}{l}\text { Peer relationship problems } \\
\text { (score } \geq 4 \text { pts) }\end{array}$ & $124(12.85)$ & $366(14.61)$ & $0.86(0.69-1.07)$ & $1.08(0.85-1.38)$ & 0.517 & 0.140 \\
\hline Prosocial behavior (score $\leq 4 \mathrm{pts}$ ) & $8(0.83)$ & $33(1.32)$ & $0.63(0.29-1.36)$ & $0.63(0.27-1.49)$ & 0.376 & 0.967 \\
\hline \multicolumn{7}{|l|}{11 years old } \\
\hline Total SDQ (score $\geq 17$ pts) & $138(14.39)$ & $342(13.75)$ & $1.06(0.85-1.30)$ & $1.19(0.94-1.50)$ & 0.157 & 0.811 \\
\hline Emotional symptoms (score $\geq 5$ pts) & $213(22.21)$ & $473(19.01)$ & $1.22(1.01-1.46)$ & $1.20(0.98-1.46)$ & 0.078 & 0.313 \\
\hline Conduct problems (score $\geq 4$ pts) & $108(11.26)$ & $343(13.79)$ & $0.79(0.63-1.00)$ & $0.93(0.72-1.20)$ & 0.561 & 0.264 \\
\hline $\begin{array}{l}\text { Hyperactivity/inattention } \\
\text { (score } \geq 7 \text { pts) }\end{array}$ & $165(17.21)$ & $391(15.72)$ & $1.12(0.91-1.36)$ & $1.20(0.96-1.49)$ & 0.112 & 0.797 \\
\hline $\begin{array}{l}\text { Peer relationship problems } \\
\text { (score } \geq 4 \text { pts) }\end{array}$ & $131(13.66)$ & $328(13.18)$ & $1.04(0.84-1.30)$ & $1.21(0.95-1.53)$ & 0.123 & 0.781 \\
\hline Prosocial behavior (score $\leq 4 \mathrm{pts}$ ) & $8(0.83)$ & $40(1.61)$ & $0.52(0.24-1.10)$ & $0.79(0.36-1.75)$ & 0.565 & 0.151 \\
\hline
\end{tabular}

${ }^{a}$ Logistic regression adjusted according to sex, maternal age, parity, national economic index, maternal educational level, smoking during pregnancy, alcohol consumption during pregnancy, maternal skin color, infection during pregnancy, pre-gestational BMI, presence of maternal mood issue and use of other analgesics during pregnancy. $\mathrm{N}$ after adjustment: $3103 .{ }^{\mathrm{b}} \mathrm{p}$-values marked in bold denote statistically significant results 
Table 3 Association between use of acetaminophen by mother during pregnancy and mental health outcomes at the age of 6 years among boys $(N=1812)$ and girls $(N=1658)$

\begin{tabular}{|c|c|c|c|c|c|}
\hline \multirow[t]{2}{*}{ Outcomes } & \multicolumn{2}{|c|}{$\begin{array}{l}\text { Frequency of outcome according to use } \\
\text { of acetaminophen during pregnancy } N(\%)\end{array}$} & \multirow{2}{*}{$\begin{array}{l}\text { Crude analysis } \\
\text { OR } \\
(95 \% \mathrm{Cl})\end{array}$} & \multicolumn{2}{|c|}{ Adjusted analysis $^{a}$} \\
\hline & $\begin{array}{l}\text { Exposed } \\
\mathrm{n}(\%)\end{array}$ & $\begin{array}{l}\text { Not exposed } \\
\mathrm{n}(\%)\end{array}$ & & $\begin{array}{l}\mathrm{OR} \\
(95 \% \mathrm{Cl})\end{array}$ & $p^{b}$ \\
\hline \multicolumn{6}{|l|}{ Boys } \\
\hline Total SDQ (score $\geq 17$ pts) & $63(12.00)$ & $149(11.58)$ & $1.04(0.76-1.43)$ & $1.28(0.90-1.83)$ & 0.163 \\
\hline Emotional symptoms (score $\geq 5$ pts) & $83(15.81)$ & $154(11.97)$ & $1.38(1.04-1.84)$ & $1.47(1.07-2.02)$ & 0.018 \\
\hline Conduct problems (score $\geq 4$ pts) & $72(13.71)$ & $223(17.33)$ & $0.76(0.57-1.02)$ & $0.93(0.68-1.27)$ & 0.641 \\
\hline Hyperactivity/inattention (score $\geq 7$ pts) & $93(17.71)$ & $195(15.15)$ & $1.21(0.92-1.58)$ & $1.42(1.06-1.92)$ & 0.020 \\
\hline Peer relationship problems (score $\geq 4$ pts) & $81(15.43)$ & $196(15.23)$ & $1.02(0.77-1.35)$ & $1.25(0.91-1.71)$ & 0.167 \\
\hline Prosocial behavior (score $\leq 4 \mathrm{pts}$ ) & $5(0.95)$ & $18(1.40)$ & $0.68(0.25-1.84)$ & $0.60(0.19-1.88)$ & 0.376 \\
\hline \multicolumn{6}{|l|}{ Girls } \\
\hline Total SDQ (score $\geq 17$ pts) & $39(8.86)$ & $122(10.02)$ & $0.87(0.60-1.28)$ & $1.02(0.67-1.54)$ & 0.943 \\
\hline Emotional symptoms (score $\geq 5$ pts) & $54(12.27)$ & $181(14.86)$ & $0.80(0.58-1.11)$ & $0.90(0.63-1.28)$ & 0.557 \\
\hline Conduct problems (score $\geq 4$ pts) & $47(10.68)$ & $173(14.20)$ & $0.72(0.51-1.02)$ & $0.79(0.54-1.16)$ & 0.226 \\
\hline Hyperactivity/inattention (score $\geq 7$ pts) & $44(10.00)$ & $149(12.23)$ & $0.80(0.56-1.14)$ & $0.76(0.51-1.12)$ & 0.165 \\
\hline Peer relationship problems (score $\geq 4$ pts) & $43(9.77)$ & $170(13.96)$ & $0.67(0.47-0.95)$ & $0.88(0.59-1.31)$ & 0.535 \\
\hline Prosocial behavior (score $\leq 4 \mathrm{pts}$ ) & $3(0.68)$ & $15(1.23)$ & $0.55(0.16-1.91)$ & $0.73(0.20-2.69)$ & 0.630 \\
\hline
\end{tabular}

a Logistic regression adjusted according to maternal age, parity, national economic index, maternal educational level, smoking during pregnancy, alcohol during pregnancy, maternal skin color, infection during pregnancy, pre-gestational BMI, presence of maternal mood disorder and use of other analgesics during pregnancy. $\mathrm{N}$ after adjustment: 1619 for boys; 1484 for girls. ${ }^{b} p$-values marked in bold denote statistically significant results

Table 4 Association between use of acetaminophen by mother during pregnancy and mental health outcomes at the age of 11 years among boys $(N=1781)$ and girls $(N=1666)$

\begin{tabular}{|c|c|c|c|c|c|}
\hline \multirow[t]{2}{*}{ Outcomes } & \multicolumn{2}{|c|}{$\begin{array}{l}\text { Frequency of outcome according to use of } \\
\text { acetaminophen during pregnancy } N(\%)\end{array}$} & \multirow{2}{*}{$\begin{array}{l}\text { Crude analysis } \\
\text { OR } \\
(95 \% \mathrm{Cl})\end{array}$} & \multicolumn{2}{|c|}{ Adjusted analysis $^{\mathrm{a}}$} \\
\hline & $\begin{array}{l}\text { Exposed } \\
\mathrm{n}(\%)\end{array}$ & $\begin{array}{l}\text { Not exposed } \\
\mathrm{n}(\%)\end{array}$ & & $\begin{array}{l}\mathrm{OR} \\
(95 \% \mathrm{Cl})\end{array}$ & $p$ \\
\hline \multicolumn{6}{|l|}{ Boys } \\
\hline Total SDQ (score $\geq 17$ pts) & $89(17.05)$ & $199(15.81)$ & $1.09(0.83-1.44)$ & $1.21(0.89-1.64)$ & 0.228 \\
\hline Emotional symptoms (score $\geq 5$ pts) & $116(22.22)$ & $223(17.71)$ & $1.33(1.03-1.71)$ & $1.31(0.99-1.73)$ & 0.052 \\
\hline Conduct problems (score $\geq 4$ pts) & $68(13.03)$ & $185(14.69)$ & $0.87(0.64-1.17)$ & $1.08(0.77-1.51)$ & 0.664 \\
\hline Hyperactivity/inattention (score $\geq 7$ pts) & $115(22.03)$ & $252(20.02)$ & $1.13(0.88-1.45)$ & $1.25(0.95-1.65)$ & 0.115 \\
\hline Peer relationship problems (score $\geq 4$ pts) & $78(14.94)$ & $191(15.17)$ & $0.98(0.74-1.31)$ & $1.16(0.85-1.59)$ & 0.339 \\
\hline Prosocial behavior (score $\leq 4$ pts) & $3(0.57)$ & $25(1.99)$ & $0.29(0.86-0.95)$ & $0.47(0.14-1.63)$ & 0.234 \\
\hline \multicolumn{6}{|l|}{ Girls } \\
\hline Total SDQ (score $\geq 17$ pts) & $49(11.21)$ & $143(11.64)$ & $0.95(0.68-1.35)$ & $1.16(0.80-1.69)$ & 0.426 \\
\hline Emotional symptoms (score $\geq 5$ pts) & $97(22.20)$ & $250(20.34)$ & $1.12(0.85-1.46)$ & $1.08(0.81-1.43)$ & 0.624 \\
\hline Conduct problems (score $\geq 4$ pts) & $40(9.15)$ & $158(12.86)$ & $0.68(0.47-0.98)$ & $0.77(0.52-1.14)$ & 0.192 \\
\hline Hyperactivity/inattention (score $\geq 7$ pts) & $50(11.44)$ & $139(11.31)$ & $1.01(0.72-1.43)$ & $1.14(0.79-1.64)$ & 0.498 \\
\hline Peer relationship problems (score $\geq 4$ pts) & $53(12.13)$ & $137(11.15)$ & $1.10(0.78-1.54)$ & $1.30(0.90-1.89)$ & 0.164 \\
\hline Prosocial behavior (score $\leq 4 \mathrm{pts}$ ) & $5(1.14)$ & $15(1.22)$ & $0.94(0.34-2.59)$ & $1.38(0.47-4.07)$ & 0.562 \\
\hline
\end{tabular}

${ }^{a}$ Logistic regression adjusted according to maternal age, parity, national economic index, maternal educational level, smoking during pregnancy, alcohol comsumption during pregnancy, maternal skin color, infection during pregnancy, pre-gestational BMl, presence of maternal mood issue and use of other analgesics during pregnancy. $\mathrm{N}$ after adjustment: 1585 boys and 1494 girls 
Table 5 Sensitivity analysis considering Edinburgh Postnatal Depression Scale at 12 months follow-up interview for the association between exposure to acetaminophen during pregnancy and SDQ total and subscales problems of children at 6 and 11 years of age

\begin{tabular}{|c|c|c|c|c|}
\hline \multirow[t]{3}{*}{ Outcomes } & \multicolumn{2}{|l|}{6 years } & \multicolumn{2}{|l|}{11 years } \\
\hline & \multicolumn{2}{|l|}{ Adjusted analysis $^{a}$} & \multicolumn{2}{|c|}{ Adjusted analysis ${ }^{a}$} \\
\hline & $\mathrm{OR}(95 \% \mathrm{Cl})$ & $p^{b}$ & $\overline{\mathrm{OR}(95 \% \mathrm{Cl})}$ & $p^{\mathrm{b}}$ \\
\hline \multicolumn{5}{|l|}{ Boys } \\
\hline Total SDQ (score $\geq 17$ pts) & $1.22(0.85-1.75)$ & 0.291 & $1.22(0.89-1.66)$ & 0.221 \\
\hline Emotional symptoms (score $\geq 5$ pts) & $1.42(1.02-1.97)$ & 0.038 & $1.33(1.01-1.76)$ & 0.045 \\
\hline Conduct problems (score $\geq 4$ pts) & $0.89(0.64-1.24)$ & 0.503 & $1.09(0.78-1.54)$ & 0.609 \\
\hline Hyperactivity/inattention (score $\geq 7$ pts) & $1.43(1.05-1.94)$ & 0.022 & $1.25(0.94-1.65)$ & 0.121 \\
\hline Peer relationship problems (score $\geq 4$ pts) & $1.21(0.88-1.67)$ & 0.251 & $1.14(0.83-1.57)$ & 0.415 \\
\hline Prosocial behavior (score $\leq 4 \mathrm{pts}$ ) & $0.65(0.21-2.03)$ & 0.457 & $0.48(0.14-1.66)$ & 0.246 \\
\hline \multicolumn{5}{|l|}{ Girls } \\
\hline Total SDQ (score $\geq 17$ pts) & $0.99(0.65-1.51)$ & 0.954 & $1.14(0.78-1.67)$ & 0.503 \\
\hline Emotional symptoms (score $\geq 5$ pts) & $0.90(0.63-1.28)$ & 0.554 & $1.06(0.79-1.42)$ & 0.694 \\
\hline Conduct problems (score $\geq 4$ pts) & $0.81(0.55-1.19)$ & 0.290 & $0.73(0.49-1.10)$ & 0.135 \\
\hline Hyperactivity/inattention (score $\geq 7$ pts) & $0.76(0.51-1.13)$ & 0.175 & $1.10(0.76-1.61)$ & 0.613 \\
\hline Peer relationship problems (score $\geq 4$ pts) & $0.89(0.60-1.32)$ & 0.559 & $1.31(0.90-1.91)$ & 0.155 \\
\hline Prosocial behavior (scor e $\leq 4 \mathrm{pts}$ ) & $0.75(0.20-2.80)$ & 0.663 & $1.39(0.47-4.15)$ & 0.553 \\
\hline
\end{tabular}

a Logistic regression adjusted according to maternal age, parity, national economic index, maternal educational level, smoking during pregnancy, alcohol consumption during pregnancy, maternal skin color, infection during pregnancy, pre-gestational BMI, presence of maternal depression and use of other analgesics during pregnancy. $\mathrm{N}$ after adjustment: 1.555 for boys and 1.137 for girls at 6 years; 1.523 for boys and 1.442 for girls at 11 years. ${ }^{b} p$-values marked in bold denote statistically significant results

\section{Discussion}

In this study, we examined the association between acetaminophen exposure during pregnancy and the behavioral outcomes of offspring in a Brazilian birth cohort. A differential effect for sex and exposure to acetaminophen was observed. In 6-year-old boys, intrauterine exposure to acetaminophen led to increased odds of having emotional and hyperactivity/inattention problems. At the age of 11 , a slight reduction in the effect size was observed. For girls, the results were nonsignificant at both follow-up times for all the considered outcomes. These findings suggest that acetaminophen exposure in the uterus may play a role in behavioral disorders in childhood, mainly in boys.

Acetaminophen is one of the most prescribed and used medications for pregnant women in Brazil [32, 33]. Comparing our results with those of previous studies, an important difference is in exposure prevalence. Although over $50 \%$ of pregnant women report using acetaminophen in the United States and Denmark [8, 34-36], we found that $27.5 \%$ of Brazilian mothers used this substance during pregnancy. We could not find Brazilian population-based studies reporting acetaminophen use during pregnancy.

Evidence from different prospective cohort studies has shown an association between acetaminophen use during pregnancy and neurobehavioral outcomes in childhood [6-12]. Three studies evaluated behavioral symptoms using the SDQ. In 7-year-old children, Liew et al. [8] observed an association between prenatal exposure to acetaminophen and higher total difficulties, conduct problems, and hyperactivity. The authors also observed increased risk when the medication was used over more than one trimester, especially near the end of the pregnancy. In addition, they found a correlation between increased frequency of use and increased risk. Stergiakouli et al. [11] demonstrated that children exposed to acetaminophen during the second and third trimesters were at higher risk of multiple behavioral difficulties, including hyperactivity and conduct problems at 7 years of age. Prenatal exposure to acetaminophen during a 32-week pregnancy was also associated with emotional symptoms. Using parent-reported SDQ scores, Thompson et al. [7] observed that acetaminophen was a risk factor for total difficulties, emotional symptoms, and conduct problems at 7 years. At age 11, however, only an association with the parent-reported emotional scale was observed.

Animal studies have supported epidemiological observational studies, providing biological plausibility for the findings. Acetaminophen is known to freely cross the placenta [37]. Recently, exposure to acetaminophen during intrauterine life was reported to affect the modulation of neurotransmission in rats [17]. Effects on neurodevelopment and behavior have also been observed in adult rats exposed to acetaminophen during intrauterine life $[15,16]$. Defects in cognitive function and deficient levels of brain-derived neurotrophic factor (BDNF) were observed in rats that 
were administered acetaminophen during neonatal life [38]. Interference in the regulation of cellular oxidative stress and the endocrine and immune systems by acetaminophen has also been suggested as a mechanism that could lead to neurodevelopment disorders [18-22, 39].

Sex-related differences in symptomatology have been reported for both ADHD and emotional problems. ADHD is more commonly diagnosed in boys than in girls [40], and girls with ADHD are more likely than boys to present inattentive ADHD and separation anxiety disorder. Boys present higher rates of hyperactivity and comorbidity for disorders such as oppositional defiant disorder and conduct disorder [40, 41]. Emotional problems in early adolescence, such as anxiety and depression, are more frequent in girls than in boys [42-45]. In this study, we tested whether sex could modify the effect of acetaminophen on behavioral problems. An interaction between acetaminophen exposure and sex was observed for the hyperactivity and emotional scales, suggesting that acetaminophen may have different effects on these problems in boys and girls. Stratified analyses suggested an effect of acetaminophen on the hyperactivity and emotional scales only in boys.

A similar effect was reported for ASD symptoms in a Spanish cohort [10]. The findings in Spain revealed that exposed boys had more ASD symptoms compared to exposed girls, who showed decreased scores. Differential acetaminophen metabolization between males and females has already been reported and may play a role in this difference, as suggested by Avella-Garcia et al. [10]. Studies on animals have demonstrated that male mice show higher toxicity levels than females after acetaminophen administration [46]. Other sex differences rely on a putatively increased vulnerability to stressors [47] and to the effect of acetaminophen on testosterone regulation $[39,48]$, neurodevelopment, and brain masculinization [15] in male brains at the beginning of life. Thus, the hypothesis that endocrine disruption influences testicular function and the production of androgens, which further affects brain development in males, could be a plausible mechanism for the present findings.

The results presented in this study suggest that the magnitude of the effect on emotional and hyperactivity/ inattention problems slightly decreased from age 6 to 11. Our sensitivity analysis using EPDS showed that the absence of an association in the adjusted analysis at the age of 11 may be due to a lack of power in the analyses for emotional problems. Our results at different ages are in line with the results reported by Thompson et al. [7], which showed differences between both ages for the parent-reported symptoms. Studies evaluating other age groups, including adolescence, are necessary to better understand the relationship between the exposure and the evaluated outcomes.
The results should be interpreted considering certain limitations. The first limitation relates to the retrospective gathering of data on medication use during pregnancy, which may have resulted in difficulty recalling acetaminophen use. Thus, the low prevalence of acetaminophen use observed in this study may be a result of underreporting. We compared our data with that collected in the same population, in 2015, by another birth cohort study (2015 Pelotas Birth Cohort). In this birth cohort, acetaminophen use information was assessed prospectively during pregnancy, as well as in the perinatal interview (retrospectively). The prevalence for use in the perinatal interview was $51 \%$ (unpublished data), which agrees with most of the data from published studies [8, 32-34]. Considering that underreporting use of acetaminophen may be a limitation for the exposure definition in our study, we expect that effect size observed in the association analyses is underestimated. Therefore, false negative results are more likely to be observed than false positives regarding this limitation. Second, both the dose-response and timing of exposure associations (higher risks for the third trimester of pregnancy exposure) had been reported previously $[8-11,13]$. However, because of possible memory issues, our data did not allow for the evaluation of exposure according to the trimester of pregnancy.

Furthermore, we cannot rule out the effect of using other medications during pregnancy on behavioral outcomes. However, other types of nonsteroidal analgesics were not associated with worse outcomes. Nonsteroidal analgesic use was also included as a covariable in the models. Thus, it is unlikely that co-medication with other analgesics influenced the findings. These results are consistent with the specificity of acetaminophen observed by Thompson et al. [7] and with the results from previous studies that used the same adjustment approach with other analgesics [8]. It has also been shown that children whose mothers have externalizing disorders (e.g., conduct disorder or drug/alcohol dependence) are at an increased risk for developing behavior problems [49]. Although we do not have information regarding externalizing problems in mothers, we included smoking and drinking behavior during pregnancy, which could be considered as proxies. Other psychological variables from mothers would also improve the robustness of our findings. Fever and inflammation during pregnancy are known risk factors for the development of neurological diseases [50]. Werenberg-Dreier et al. [51] showed that exposure to fever and infection over specific timeframes during pregnancy was associated with the occurrence of ADHD. Although the women in the present study reported using the medication, their reasons for doing so were not available. This information would also be important to improve the robustness of our findings. However, this information was not collected in 2004. 
Another critical limitation, which has been greatly discussed in the literature, was the presence of residual confounding due to socioeconomic variables. Additional file 1: Table S1 shows socioeconomic variables, including educational level, economic index, skin color, and parity, which are variables that behave as proxies for socioeconomic position (SEP) in Brazil. These variables were all associated with the use of acetaminophen during pregnancy and with most of the outcomes at different ages, especially hyperactivity/inattention problems. While other variables were not statistically associated with our main exposure, we included some of them in the adjusted models given recent evidence suggesting them as possible confounders of this association. However, these variables might not be as important as SEP variables in our models. It is unlikely that residual confounding due to a lack of control over other SEP measurements, such as income and work, may have biased our results. Models were adjusted for variables associated with exposure. Further, the results considered most of the effects of SEP. In addition, crude and adjusted measures of the association between acetaminophen exposure and emotional problems at 11 years of age are very similar, reinforcing the idea that the association was, probably, not confounded by these variables.

\section{Conclusions}

The use of acetaminophen in pregnant mothers was associated with subsequent emotional and hyperactivity symptoms in their 6-year-old boys. A suggestive association was observed in 11-year-old boys. Further studies that gather more precise information concerning dose and time of exposure among pregnant women are necessary to establish the long-term effects of maternal acetaminophen use during pregnancy on offspring.

\section{Additional file}

Additional file 1: Table S1. Association analysis between covariates, exposure and relevant outcomes. (DOCX $22 \mathrm{~kb}$ )

\section{Acknowledgements}

Not applicable

\section{Funding}

This article is based on data from the study "Pelotas Birth Cohort, 2004" conducted by Postgraduate Program in Epidemiology at Universidade Federal de Pelotas, with the collaboration of the Brazilian Public Health Association (ABRASCO). From 2009 to 2013, the Wellcome Trust supported the 2004 birth cohort study. The World Health Organization, National Support Program for Centers of Excellence (PRONEX), Brazilian National Research Council (CNPq), Brazilian Ministry of Health, and Children's Pastorate supported previous phases of the study. The 11-year follow-up was supported by the Science and Technology Department / Brazilian Ministry of Health, with resources transferred through the Brazilian National Council for Scientific and Technological Development (CNPq), grant 400943/2013-1. This article is supported by São Paulo Reseach Foundation - FAPESP (grant number 2014/13864-6). This study was financed in part by the Coordenação de Aperfeiçoamento de Pessoal de Nível Superior - Brasil (CAPES) - Finance Code 001. Alicia Matijasevich and Iná S. Santos are supported by the CNPq (National Council for Scientific and Technological Development).

\section{Availability of data and materials}

The datasets used and analyzed during the current study are available from the corresponding author on reasonable request.

\section{Authors' contributions}

LT-R, BCS, BD-P, LE, CLdM, ADB, TM-S, FSLV, TNM conceived the work, conducted the analyses and draft the first version of the manuscript. ISS, AM, AB made substantial contributions to acquisition and interpretation of data. MFS, ISS, AM, AB, LAR, LS-F, ADB revised the manuscript critically and contributed with interpretation of the findings. All authors gave final approval of the version to be published.

\section{Ethics approval and consent to participate}

The Medical Ethics Committee of the Federal University of Pelotas approved the study protocol and all follow-ups of the Pelotas 2004 Birth Cohort Study. Mothers signed a consent form on behalf of them and their children at each follow-up, after being informed of the study objectives. At the 11-year follow-up, the cohort participants aged 11 years or more also signed an informed consent form.

\section{Consent for publication}

Not applicable

\section{Competing interests}

LAR has been a member of the speakers' bureau/advisory board and/or acted as a consultant for Eli-Lilly, Janssen-Cilag, Medice, Novartis and Shire in the last three years. He receives authorship royalties from Oxford Press and ArtMed. He has also received travel awards from Shire for his participation in the 2018 APA meetings and from Novartis to take part of the 2016 AACAP meeting. The ADHD and Juvenile Bipolar Disorder Outpatient Programs chaired by him received unrestricted educational and research support from the following pharmaceutical companies in the last three years: Janssen -

Cilag, Novartis, and Shire.

\section{Publisher's Note}

Springer Nature remains neutral with regard to jurisdictional claims in published maps and institutional affiliations.

\section{Author details}

${ }^{1}$ Postgraduate Program in Epidemiology, Universidade Federal de Pelotas, Rua Marechal Deodoro, 1160 - $3^{\circ}$ andar, Pelotas, RS 96020-220, Brazil. ${ }^{2}$ School of Nursing and Public Health, Universidade Federal de Pelotas, Pelotas, Brazil. ${ }^{3}$ Hospital de Clínicas de Porto Alegre, Porto Alegre, Brazil. ${ }^{4}$ Postgraduate Program in Genetics and Molecular Biology, Universidade Federal do Rio Grande Do Sul, Porto Alegre, Brazil. ${ }^{5}$ Genomic Medicine Laboratory, Hospital de Clínicas de Porto Alegre, Porto Alegre, Brazil. ${ }^{6}$ Laboratory of Research in Bioethics and Ethics in Research, Hospital de Clínicas de Porto Alegre, Porto Alegre, Brazil. ${ }^{7}$ Department of Psychology, School of Medicine, Universidade Federal de Pelotas, Pelotas, Brazil. ${ }^{8}$ Departamento de Medicina Preventiva, Faculdade de Medicina FMUSP, Universidade de São Paulo, São Paulo, Brazil. ${ }^{9}$ Department of Psychiatry, Hospital de Clínicas de Porto Alegre, Universidade Federal do Rio Grande do Sul, Porto Alegre, Brazil. ${ }^{10}$ National Institute of Developmental Psychiatry for Children and Adolescents, São Paulo, Brazil.

Received: 5 July 2018 Accepted: 25 October 2018

Published online: 20 November 2018

\section{References}

1. Brune K, Renner B, Tiegs G. Acetaminophen/paracetamol: A history of errors, failures and false decisions. Eur J Pain (London, England). 2015;19(7):953-65 Epub 2014/11/29.

2. de Fays L, Van Malderen K, De Smet K, Sawchik J, Verlinden V, Hamdani J, et al. Use of paracetamol during pregnancy and child neurological development. Dev Med Child Neurol. 2015;57(8):718-24 Epub 2015/04/09.

3. Mengue SS, Schenkel EP, Duncan BB, Schmidt MI. Drug use by pregnant women in six Brazilian cities. Rev Saude Publica. 2001:35(5):415-20 Epub 2001/11/28. Uso de medicamentos por gestantes em seis cidades brasileiras.

4. Guerra GCB, da Silva AQB, França LB, Assunção PMC, Cabral RX, Ferreira AAA. Drug use during pregnancy in Natal, Brazil. Rev Bras Ginecol Obstet. 2008;30(1):12-8 
5. Melo SCCS, Pelloso SM, Carvalho MDB, Oliveira NLB. Uso de medicamentos por gestantes usuárias do Sistema Único de Saúde. Acta Paul Enferm. 2009; 22:66-70.

6. Liew Z, Ritz B, Virk J, Olsen J. Maternal use of acetaminophen during pregnancy and risk of autism spectrum disorders in childhood: a Danish national birth cohort study. Autism Res. 2016;9(9):951-8.

7. Thompson JMD, Waldie KE, Wall CR, Murphy R, Mitchell EA, the ABCsg Associations between Acetaminophen Use during Pregnancy and ADHD Symptoms Measured at Ages 7 and 11 Years. PLoS One. 2014;9(9):e108210 Epub 2014/09/25.

8. Liew Z, Ritz B, Rebordosa C, Lee PC, Olsen J. Acetaminophen use during pregnancy, behavioral problems, and hyperkinetic disorders. JAMA Pediatr. 2014;168(4):313-20 Epub 2014/02/26.

9. Brandlistuen RE, Ystrom E, Nulman I, Koren G, Nordeng H. Prenatal paracetamol exposure and child neurodevelopment: a sibling-controlled cohort study. Int J Epidemiol. 2013;42(6):1702-13 Epub 2013/10/29.

10. Avella-Garcia CB, Julvez J, Fortuny J, Rebordosa C, Garcia-Esteban R, Galan $\mid \mathrm{R}$, et al. Acetaminophen use in pregnancy and neurodevelopment: attention function and autism spectrum symptoms. Int J Epidemiol. 2016; 45(6):1987-96 Epub 2016/06/30.

11. Stergiakouli E, Thapar A, Davey SG. Association of Acetaminophen use during Pregnancy with Behavioral Problems in childhood: evidence against confounding. JAMA Pediatr. 2016;170(10):964-70 Epub 2016/08/18.

12. Ystrom E, Gustavson K, Brandlistuen RE, Knudsen GP, Magnus P, Susser E, et al. Prenatal Exposure to Acetaminophen and Risk of ADHD. Pediatrics. 2017; 140(5) Epub 2017/11/01

13. Vlenterie R, Wood ME, Brandlistuen RE, Roeleveld N, van Gelder MM, Nordeng $\mathrm{H}$. Neurodevelopmental problems at 18 months among children exposed to paracetamol in utero: a propensity score matched cohort study. Int J Epidemiol. 2016:45(6):1998-2008 Epub 2016/09/03.

14. Masarwa R, Levine H, Gorelik E, Reif S, Perlman A, Matok I. Prenatal exposure to acetaminophen and risk for attention deficit hyperactivity disorder and autistic Spectrum disorder: a systematic review, meta-analysis, and meta-regression analysis of cohort studies. Am J Epidemiol. 2018; Epub 2018/04/25.

15. Hay-Schmidt A, Finkielman OTE, Jensen BAH, Hogsbro CF, Bak Holm J, Johansen $\mathrm{KH}$, et al. Prenatal exposure to paracetamol/acetaminophen and precursor aniline impairs masculinisation of male brain and behaviour. Reproduction (Cambridge, England). 2017;154(2):145-52 Epub 2017/06/01.

16. Philippot G, Gordh T, Fredriksson A, Viberg H. Adult neurobehavioral alterations in male and female mice following developmental exposure to paracetamol (acetaminophen): characterization of a critical period. J Appl Toxicol. 2017;37(10):1174-81 Epub 2017/04/28.

17. Blecharz-Klin K, Joniec-Maciejak I, Jawna K, Pyrzanowska J, Piechal A, Wawer A, et al. Developmental exposure to paracetamol causes biochemical alterations in medulla oblongata. Environ Toxicol Pharmacol. 2015;40(2): 369-74 Epub 2015/08/04

18. Shaheen SO, Newson RB, Ring SM, Rose-Zerilli MJ, Holloway JW, Henderson AJ. Prenatal and infant acetaminophen exposure, antioxidant gene polymorphisms and childhood asthma. J Allergy Clin Immunol. 2010;126(6): $1141-8$

19. Nuttall SL, Khan JN, Thorpe GH, Langford N, Kendall MJ. The impact of therapeutic doses of paracetamol on serum total antioxidant capacity. J Clin Pharm Ther. 2003;28(4):289-94 Epub 2003/08/13.

20. Thiele K, Solano ME, Huber S, Flavell RA, Kessler T, Barikbin R, et al. Prenatal acetaminophen affects maternal immune and endocrine adaptation to pregnancy, induces placental damage, and impairs fetal development in mice. Am J Pathol. 2015;185(10):2805-18 Epub 2015/08/09.

21. Kristensen DM, Lesne L, Le Fol V, Desdoits-Lethimonier C, Dejucq-Rainsford $\mathrm{N}$, Leffers $\mathrm{H}$, et al. Paracetamol (acetaminophen), aspirin (acetylsalicylic acid) and indomethacin are anti-androgenic in the rat foetal testis. Int J Epidemiol. 2012:35(3):377-84 Epub 2012/05/23.

22. Albert O, Desdoits-Lethimonier C, Lesne L, Legrand A, Guille F, Bensalah K, et al. Paracetamol, aspirin and indomethacin display endocrine disrupting properties in the adult human testis in vitro. Hum Reprod (Oxford, England) 2013;28(7):1890-8 Epub 2013/05/15

23. Brion MJ, Victora C, Matijasevich A, Horta B, Anselmi L, Steer C, et al. Maternal smoking and child psychological problems: disentangling causal and noncausal effects. Pediatrics. 2010;126(1):e57-65 Epub 2010/07/01.

24. Brion MJ, Lawlor DA, Matijasevich A, Horta B, Anselmi L, Araujo CL, et al. What are the causal effects of breastfeeding on $I Q$, obesity and blood pressure? Evidence from comparing high-income with middle-income cohorts. Int J Epidemiol. 2011:40(3):670-80 Epub 2011/02/26

25. Murray E, Pearson R, Fernandes M, Santos IS, Barros FC, Victora CG, et al. Are fetal growth impairment and preterm birth causally related to child attention problems and ADHD? Evidence from a comparison between high-income and middle-income cohorts. J Epidemiol Community Health. 2016;70(7):704-9 Epub 2016/01/16.

26. Santos IS, Barros AJD, Matijasevich A, Zanini R, Chrestani Cesar MA, Camargo-Figuera FA, et al. Cohort Profile Update: 2004 Pelotas (Brazil) Birth Cohort Study. Body composition, mental health and genetic assessment at the 6 years follow-up. Int J Epidemiol. 2014;43(5):1437.

27. Santos IS, Barros AJ, Matijasevich A, Domingues MR, Barros FC, Victora CG. Cohort profile: the 2004 Pelotas (Brazil) birth cohort study. Int J Epidemiol. 2011;40(6):1461-8 Epub 2010/08/13.

28. Fleitlich-Bilyk B, Goodman R. Prevalence of child and adolescent psychiatric disorders in Southeast Brazil. J Am Acad Child Adolesc Psychiatry. 2004; 43(6):727-34 Epub 2004/05/29.

29. Barros AJD, Victora CG. Indicador econômico para o Brasil baseado no censo demográfico de 2000. Rev Saude Publica. 2005;39(4):523-9.

30. Cox JL, Holden JM, Sagovsky R. Detection of postnatal depression. Development of the 10-item Edinburgh postnatal depression scale. $\mathrm{Br}$ Psychiatry J Ment Sci. 1987;150:782-6 Epub 1987/06/01.

31. Santos IS, Matijasevich A, Tavares BF, Barros AJD, Botelho IP, Lapolli C, et al. Validation of the Edinburgh postnatal depression scale (EPDS) in a sample of mothers from the 2004 Pelotas birth cohort study. Cad Saude Publica. 2007:23:2577-88.

32. Costa DB, Coelho HLL, Santos DB. Utilização de medicamentos antes e durante a gestação: prevalência e fatores associados. Cad Saude Publica. 2017;33:e00126215.

33. Brum LFS, Pereira P, Felicetti LL, Silveira RD. Utilização de medicamentos por gestantes usuárias do Sistema Único de Saúde no município de Santa Rosa (RS, Brasil). Cien Saude Colet. 2011;16:2435-42.

34. Werler MM, Mitchell AA, Hernandez-Diaz S, Honein MA. Use of over-thecounter medications during pregnancy. Am J Obstet Gynecol. 2005;193(3 Pt 1):771-7 Epub 2005/09/10

35. Rebordosa C, Kogevinas M, Bech BH, Sorensen HT, Olsen J. Use of acetaminophen during pregnancy and risk of adverse pregnancy outcomes. Int J Epidemiol. 2009:38(3):706-14 Epub 2009/04/01.

36. Jensen MS, Rebordosa C, Thulstrup AM, Toft G, Sorensen HT, Bonde JP, et al. Maternal use of acetaminophen, ibuprofen, and acetylsalicylic acid during pregnancy and risk of cryptorchidism. Epidemiology (Cambridge, Mass). 2010;21(6):779-85 Epub 2010/09/02.

37. Levy G, Garrettson LK, Soda DM. Evidence of placental transfer of acetaminophen. Pediatrics. 1975;55(6):895 Epub 1975/06/01.

38. Viberg H, Eriksson P, Gordh T, Fredriksson A. Paracetamol (acetaminophen) administration during neonatal brain development affects cognitive function and alters its analgesic and anxiolytic response in adult male mice. Toxicol Sci. 2014;138(1):139-47 Epub 2013/12/24

39. van den Driesche S, Macdonald J, Anderson RA, Johnston ZC, Chetty T, Smith LB, et al. Prolonged exposure to acetaminophen reduces testosterone production by the human fetal testis in a xenograft model. Sci Transl Med. 2015;7(288):280-8 Epub 2015/05/23.

40. Dalsgaard S, Mortensen PB, Frydenberg M, Thomsen PH. Conduct problems, gender and adult psychiatric outcome of children with attention-deficit hyperactivity disorder. Br J Psychiatry. 2002;181(5):416-21 Epub 2018/01/02.

41. Levy F, Hay DA, Bennett KS, McStephen M. Gender differences in ADHD subtype comorbidity. J Am Acad Child Adolesc Psychiatry. 2005;44(4):36876 Epub 2005/03/23.

42. Bakhla AK, Sinha P, Sharan R, Binay Y, Verma V, Chaudhury S. Anxiety in school students: role of parenting and gender. Ind Psychiatry J. 2013;22(2): 131-7 Epub 2014/07/12

43. Costello EJ, Mustillo S, Erkanli A, Keeler G, Angold A. Prevalence and development of psychiatric disorders in childhood and adolescence. Arch Gen Psychiatry. 2003;60(8):837-44 Epub 2003/08/13

44. Poulton R, Milne BJ, Craske MG, Menzies RG. A longitudinal study of the etiology of separation anxiety. Behav Res Ther. 2001;39(12):1395-410 Epub 2002/01/05

45. Weiss DD, Last CG. The developmental psychopathology of anxiety. Developmental variations in the prevalence and manifestation of anxiety disorders; 2015.

46. McConnachie LA, Mohar I, Hudson FN, Ware CB, Ladiges WC, Fernandez C, et al. Glutamate cysteine ligase modifier subunit deficiency and gender as 
determinants of acetaminophen-induced hepatotoxicity in mice. Toxicol Sci. 2007:99(2):628-36.

47. Taylor E. In: Rutter M, Bishop D, Pine D, Scott S, Stevenson JS, Thapar A, editors. Rutter's child and adolescent psychiatry. 5th ed. Oxford: WILEYBLACKWELL; 2010

48. Kristensen DM, Hass U, Lesne L, Lottrup G, Jacobsen PR, DesdoitsLethimonier $\mathrm{C}$, et al. Intrauterine exposure to mild analgesics is a risk factor for development of male reproductive disorders in human and rat. Hum Reprod (Oxford, England). 2011;26(1):235-44 Epub 2010/11/10.

49. Weijers D, van Steensel FJA, Bogels SM. Associations between psychopathology in mothers, fathers and their children: a structural modeling approach. J Child Fam Stud. 2018;27(6):1992-2003 Epub 2018/05/15

50. Mann JR, McDermott $S$. Are maternal genitourinary infection and preeclampsia associated with ADHD in school-aged children? J Atten Disord. 2010:15(8):667-73

51. Werenberg Dreier J, Nybo Andersen AM, Hvolby A, Garne E, Kragh Andersen P, Berg-Beckhoff G. Fever and infections in pregnancy and risk of attention deficit/hyperactivity disorder in the offspring. J Child Psychol Psychiatry Allied Discip. 2016;57(4):540-8 Epub 2015/11/05

Ready to submit your research? Choose BMC and benefit from:

- fast, convenient online submission

- thorough peer review by experienced researchers in your field

- rapid publication on acceptance

- support for research data, including large and complex data types

- gold Open Access which fosters wider collaboration and increased citations

- maximum visibility for your research: over $100 \mathrm{M}$ website views per year

At $\mathrm{BMC}$, research is always in progress.

Learn more biomedcentral.com/submissions 